\title{
Historia social de la educación en ciencias en Colombia: la segunda mitad del siglo XX
}

Social history of the education in sciences in Colombia: Second half of century XX

Royman Pérez Miranda1, Rómulo Gallego Badillo', Adriana Patricia Gallego Torres ${ }^{2}$ y Roberto Enrique Figueroa Molina ${ }^{3}$

${ }^{1}$ Profesores investigadores de la Universidad Pedagógica Nacional de Bogotá, Colombia. ${ }^{2}$ Profesora investigadora de la Universidad Distrital Francisco José de Caldas, Bogotá, Colombia. ${ }^{3}$ Profesor investigador de la Universidad del Atlántico, Barranquilla, Colombia. royman@pedagogica.edu.co rgallego@pedaggoica.edu.co adpgallegot@udistrita.edu.co roberfigue@hotmail.com

\section{Resumen}

Con esta ponencia se presentan los fundamentos, desarrollo y resultados parciales de un proyecto de investigación sobre la historia social de la educación en ciencias en Colombia durante la segunda mitas del siglo XX, proyecto patrocinado por COLCIENCIAS y bajo la responsabilidad de los grupos de investigación, IREC, de la Universidad Pedagógica Nacional, de Bogotá, D. C., Colombia, y GECIT de la Universidad del Atlántico, de Barranquilla, Colombia.

\section{Palabras clave}

Didáctica de las ciencias; Historia social de las ciencias; ciencia, tecnología y sociedad.

\section{Abstract}

With this communication the foundations appear, development and partial results of an investigation project on the social history of the education in sciences in Colombia during the second half of century XX, project sponsored by COLCIENCIAS and under the responsibility of the investigation groups, IREC, of the National Pedagogical University, of Bogotá, D. C., Colombia, and GECIT of the Atlantic University of Barranquilla, Colombia

\section{Key words}

Didactics of sciences; Social history of sciences; science, technology and society

\section{Introducción}

Este proyecto de investigación está patrocinado por COLCIENCIAS y por las tres universidades a las que pertenecen los investigadores. Se inscribe en la historia social de las ciencias, en este caso de Colombia, puesto que los responsables son del parecer de que la historia social de la educación en ciencias hace parte de esa historia (Bowles y Morus, 2007). En consecuencia se ha centrado en el análisis de los planes de estudio de los programas de Licenciatura en Ciencias, la historia legal de los de las escuelas normales desde 1950 hasta 1999, como también del análisis cuítico de los contextos sociales, culturales, político y económicos de esa segunda mitad del siglo XX.

Metodológicamente además, se realizan entrevistas a egresados y directores de los programas de licenciatura, como a los rectores y profesores de ciencias de las actuales Escuelas Normales Superiores. Durante 2008 el trabajo se concentró en estas últimas entrevistas y se ha planeado que durante 2009 los investigadores se ocuparan principalmente de los programas de licenciatura en 
ciencias. Es esta a perspectiva de las concepciones científicas dada por la dinámica de los sistemas no lineales de las relacione CTSAC; que en este texto se hace una propuesta.

\section{Fundamentos conceptuales}

Estos son prinapalmente histórico-epistemológicos y didácticos, como se especifican a continuación.

Las Propuestas Histórico Epistemológicas. El siglo XX se inicia con el convencimiento de que hay una ciencia: la física; con una filosofía: el positivismo, basado en la lógica inductiva, del que se deriva el denominado método cient́fico; y la aceptación de la mirada determinista. Estas convicciones harán crisis entre 1905, y 1926. En 1905 con la publicación de los famosos tres artículos de A. Einstein; en 1925 con el de W. Heisemberg; y en 1926 con el de E. Schrödinger. Se produce entonces una revolución en el seno de la física que le pone límites al determinismo con la visión probabilista de la mecánica cuántica.

El siglo XX comienza también con la paulatina consolidación de la comunidad de historiadores de las ciencias (Barona, 1994). En 1938 se inicia la historia social de la ciencia con el primer trabajo de R. K. Merton, "Science, technology, and society in seventeen century England' y luego se consolida con su segunda investigación "Theory and social structure", de 1949. En ellos, Merton demuestra que en este contexto espećfico, la ciencia se desarrolla debido a una cultura particular que contribuyó a la institucionalización de la actividad cient́fica y debido también a las relaciones que se establecieron entre ciencia, técnica y poder político y militar. Ese origen y desarrollo de la ciencia, se hallan estrechamente ligados al origen de la sociedad modema y a los valores, intereses y estructuras básicas del capitalismo industrial, con la adopción de los valores morales y utilitarios del puritanismo. Afirmación esta que es objeto de discusión (Bowler y Rhysmorus, 2007). Comienzan a estudiarse entonces las relaciones entre ciencia, tecnología y sociedad (CTS) (Vessuri, 1992; Restivo, 1992).

La propuesta de T. S. Kuhn (1972) de 1962 y los dos artíaulos de I. Lakatos (1983) de 1970 y 1972, reorientaron las investigaciones sobre la historia interna de las ciencias. A finales del siglo XX, hubo acuerdos de que las investigaciones histónicas "internalistas" y las "externalistas" eran complementarias. Se adoptó la taxonomía de modelo icónico o gráfico, modelo analógico y modelo simbólico (Hanson, 1977; Gieré, 1990; Lombardi, 1998; Tomasi, 1999; Caldin, 2002). Las décadas del sesenta y del setenta del siglo XX revolucionaron las concepciones que sobre ciencia e historia de la ciencia se habían construido en los comienzos del mismo siglo (Echeverría, 1998). De la misma manera, se reafirmó la existencia de una pluralidad de reconstrucciones históricas (Estany, 2005), igualmente, que no había, un esquema único para juzgar la cientificidad de cualquier elaboración conceptual y metodológica, a la vez que quedó en daro la también pluralidad de ciencias, cada una de ellas con su propio lenguaje y colectivos de especialistas con sus objetos de conocimiento y sus estructuras conceptuales y metodológicas características.

\section{De la didáctica de las ciencias}

Hay que poner también de presente que en el siglo $X X$, en Colombia la didáctica era el componente instrumental de la pedagogía, considerada esta última como la ciencia de la educación. En el país, de conformidad con los resultados de un proyecto de investigación sobre la formación inicial de profesores de ciencias en la Esauela Normal Superior de Colombia (Gallego Badillo, Pérez Miranda y Rincón Pabón, 2008). En virtud de que estaba oficialmente prohibida aualquier discusión pública de los fundamentos del positivismo, la aplicación sotenada del Método Cientúico, contribuyó a la divulgación en las aulas de una epistemología ingenua (Gallego Badillo, 
Pérez Miranda y Rincón Pabón, 2008). Esta fue la versión de ciencia en la que se formaron los profesores para la enseñanza secundaria y la que estos socializaron entre el estudiantado, incluso hasta finales del siglo XX (Gallego Badillo, Pérez Miranda, Torres de Gallego y Amador Rodríguez, 2004; Gallego Badillo, Pérez Miranda y Torres de Gallego, 2004).

A partir de los años ochenta del siglo pasado, se multiplicaron las revistas especializadas e indexadas en las que se pusieron en circulación los resultados de las investigaciones didácticas y la propuesta de modelos cient́ficos para esta disciplina. Se consolidó la comunidad de didactas de las distintas ciencias de la naturaleza, se formularon y desarrollaron los campos de saber y de investigación desde los problemas de saber que los ha caracterizado (Gil, Carrascosa y MartínezTerrades, 1991). Se incrementaron los congresos nacionales, regionales e internacionales. Contar con todos estos elementos caracteństicos de una ciencia determinada, habló en favor del estatuto cientúfico de la didáctica de las ciencias.

En la actualidad se halla en la mesa de discusión el estatuto epistemológico de la didáctica de las ciencias (Adúriz-Bravo 2000; Adúriz-Bravo e Izquierdo Aymerich, 2001; Adúriz-Bravo e Izquierdo Aymerich, 2002; Gallego Badillo, 2004; Gallego Torres y Gallego Badillo, 2006). La discusión continuará por parte de la comunidad de especialistas, una actitud necesaria y de vigilancia que ha de darse en el interior de aralquier disciplina cuyos integrantes son concientes de que no construyen verdades absolutas, y mucho menos cuando esa disciplina tiene que ver con los seres humanos, afectada por las condiciones culturales, políticas y económicas.

En cuanto a los campos de saber y de investigación formulados por la comunidad de didactas de las ciencias, cabe mencionar aquí el de la formación inicial y continua de profesores de ciencias; el de las relaciones entre ciencia, historia y didáctica de las ciencias; el de la confiabilidad de los textos de enseñanza cuyos resultados han demostrado que esos textos transmiten errores conceptuales, una versión de ciencia de carácter positivista, descontextualizada y ahistórica, reducida a ser una ciencia producto limitada a definiciones de conceptos y a algoritmos para la solución de ejercicios de lápiz y papel; y, el del uso de las TICs en la educación en ciencias; el de las concepciones epistemológicas didácticas y pedagógicas de los profesores de ciencias; y el de las concepciones altemativas del estudiantado (Furió, 1996; Pozo, 1996), que se inició con las investigaciones de las concepciones enradas de los estudiantes y que en la actualidad se ha desplazado hacia el problema de los modelos mentales (Gutiérrez, 2005).

Otro campo es el de la conversión en ciencia escolar de la ciencia que practican los científicos, a través del proceso de transposición (Chevallard, 1985) o de recontextualización didáctica contenida en los textos de enseñanza. Las investigaciones adelantadas parecen demostrar que muchos de los textos analizados contienen tergiversaciones conceptuales, a la vez que no tratan el problema de la historia e imponen una aproximación epistemológica empiropositivista (Níaz, 1998; De la Gandara, Gil, y Sanmartí, 2002; Cuellar, 2004; Cuellar, Gallego Badillo y Pérez Miranda, 2008).

Estudios realizados permiten afirmar que la concepción de ciencia que se transmite en la educación colombiana sigue siendo la empiropositivista, en la que la formulación de los modelos científicos ha sido labor de individuos geniales (Gallego Torres, 2002), independientes y aislados de las condiciones culturales, políticas y económicas del tiempo en que desarrollaron su labor. Desde esta perspectiva, que no hace referencia a la historia social de las ciencias, no se suele analizar el sistema educativo dentro del cual se formaron esos genios (Toulmin, 1977). Son estas imágenes de cientúfico y de actividad cient́fica que imponen habitualmente los textos de enseñanza. 


\section{De los textos de enseñanza}

Los textos de enseñanza transmiten una versión de ciencia positivista y ahistórica, como también de manera tácita o explícita, una concepción didáctica y pedagógica de la ciencia de la cual dan auenta en sus contenidos. Cada texto didáctico o libro de enseñanza es Alcoy: en sí y en el mejor de los caso, una interpretación de los autores de los originales en los que la comunidad científica sometió su propuesta de modelo científico a sus pares académicos, en la correspondiente revista especializada. Pero hay igualmente, textos de enseñanza que son copias de estos, por lo que se constituyen en la versión de la versión de esos originales. Además, suelen no hacer citas ni presentar referencias bibliográficas, con lo que dejan la impresión de un conocimiento científico verdadero e indiscutible, todo en razón de que transmiten una ciencia producto y no como actividad contextualizada.

Así, habría que sostener que la enseñanza de las ciencias basada en el seguimiento de estos textos, se remite a una transmisión de contenidos transpuestos que, en la mayoría de los casos, no refiejan la historia interna y externa de la actividad y de la cultura científica, en cuyo seno se produjeron los modelos cient́ficos. Como han demostrado las investigaciones didácticas en este campo sobre los errores conceptuales y metodológicos, histórica, epistemológica, didáctica y pedagógicamente intencionados, transmiten, para reiterarlo, una versión de ciencia y de actividad científica acorde con la creación de una comunidad de usuarios de los productos de las investigaciones cientúfico-tecnológicas.

\section{Metodología}

El grupo interinstitucional responsable de esta investigación se propuso realizar un estudio sistemático de las concepciones de Historia, de Epistemología, de Didáctica y de Pedagogía que orientaron la formulación de los currículos para la formación de profesores de ciencias para el nivel medio, y de normalistas para el nivel básico del sistema educativo colombiano, durante la segunda mitad del siglo $X X$ y determinar las relaciones de estos programas y planes de estudio con el entorno social, cultural, político y económico del país en ese período así como analizar los cambios introducidos en esos programas en el lapso delimitado.

Para lograr esos objetivos se asumió la metodología de la investigación documental de carácter cualitativo-interpretativo que se inscribe en las categorías de confiabilidad y validez de las nuevas elaboraciones epistemológicas pospositivistas (Martínez, 2006). Se centra en los registros históricos de los currículos que se formularon y modificaron a lo largo de la segunda mitad del siglo XX, en las escuelas normales, en lo que a la educación en ciencias se refiere y los marcos legales en los que se inscribieron, acreditaron y/o recibieron registros calificados. El procedimiento que se siguió, fue la selección de las instituciones educativas, la recolección de los documentos y la elaboración y validación de cinco matrices para los correspondientes análisis de los documentos (Gallego Badillo, Pérez Miranda y Torres de Gallego, 2004; Gallego Badillo, Pérez Miranda, Torres de Gallego y Amador Rodríguez, 2004); así, una para lo histórico, otra para lo epistemológico, la tercera para lo didáctico, la cuarta para lo pedagógico y la quinta para interpretar el contexto sociopolítico y económico.

De conformidad con lo anotado se formularon los siguientes interrogantes: ¿Qué versión de ciencia se ha socializado a través de la educación en ciencias en Colombia, en la segunda mitad del siglo XX? ¿Qué tratamiento de la historia de las ciencias y de la historia social de las ciencias se ha hecho objeto de trabajo en las aulas? ¿Desde qué didáctica se ha realizado ese trabajo? ¿Qué incidencia significativa han tenido los cambios culturales, sociales y políticos del país en los proyectos curriculares de esa segunda mitad del siglo XX? 
Además, con base en un análisis hermenéutico de los documentos legales sobre educación y de los currículos de los programas académicos para la formación inicial de profesores de ciencias, para la formación de normalistas y para la educación en ciencias en los niveles básico y medio de la educación colombiana: ¿Es factible elaborar una historia social de la educación en ciencias en el país? ¿Hasta dónde las lecturas basadas en las nuevas aproximaciones histórico-epistemológicas, didácticas y pedagógicas constituyen un anacronismo? (Amold, 2003).

\section{Resultados}

Por razones de planeación los responsables de esa investigación decidieron trabajar en la primera fase (2008), las actualmente denominadas Escuelas Normales Superiores", con sus cidos complementarios. La segunda fase (2009) se dedicará a las Facultades de Educación. Con base en las matrices y los criterios de análisis de la documentación revisada y referida a las Escuelas Normales y las políticas de formación, se establecieron las siguientes.

\section{Consideraciones preliminares}

Identificada y caracterizada la historia de las Escuelas Normales en la investigación y teniendo en arenta que en estas instituciones se forman los profesores que tienen la labor profesional de iniciar entre los educandos la socialización de la actividad y la cultura científicas, habría que sostener que:

$>$ Los PEI parecen seguir un modelo impuesto por los especialistas del Ministerio de Educación Nacional (MEN), a un cuando se les otorgó cierta autonomía las Escuelas Normales, para que desde su trayectoria y fortalezas académicas decidieran el área de énfasis y las concepciones pedagógicas decidieran la estructuración de su propio proyecto. Un análisis de cada una de las historias consignadas en los PEI, muestra como la mayoría de ellas se dedican a una especie de elogio a lo realizado en el tiempo. Solo dos de ellas hacen reconstrucción crítica, señalando el control centralizado a que han estado sometidas estas instituciones formadoras de maestros.

$>$ Preocupan las referencias que rectores o coordinadores académicos hacen de lo sucedido con los convenios que cada una de estas Escuelas Normales Superiores establecieron con las universidades para el proceso de acreditación, por cuanto parece haberse remitido a términos meramente económicos, por fuera de un proyecto, en este caso, de una socialización de las concepciones y de la actividad científica entre la población escolar de educación básica y el desarrollo de la pedagogía y la didáctica de las ciencias de la naturaleza; algo que en principio habla de las intencionalidades formativas de esas universidades y sus Facultades de Educación, en cuanto a la formación de profesores de ciencias.

$>$ Con algunas excepciones destacables, la enseñanza de las ciencias parece seguir remitida a una versión que las asume como producto dentro de una aproximación positivista que las asume como verdades absolutas. Hay que reconocer sin embargo y en este contexto, ciertas tendencias en las que se intenta superar el paradigma de la transmisión verbal de contenidos curriculares.

$>\quad$ No hay aún un convencimiento de que cada una de las ciencias de la naturaleza que se hace objeto de enseñanza, posee una historia de construcción ligada a la cultura y a los proyectos políticos y económicos de aquellas naciones que le apostaron a la investigación científicotecnológica y que en la actualidad son las que detentan el poder y la decisión acerca del destino de la humanidad. En otras palabras, la ciencia que se hace objeto de enseñanza en las Escuelas Normales Superiores (ENS) auscultadas es descontextualizada, por lo que se socializa que aquello que esas ciencias afirman se encuentra "escondido" en la naturaleza. Cuando en algunas de estas instituciones los profesores se ocupan de ella, se centran únicamente en la biografía de esos personajes que fueron autores de las teorías. No hay todavía una aproximación destacable a la historia social de las ciencias. 
> Hay una concepción sin fundamentos histórico-epistemológicos sobre las relaciones entre cada una de las ciencias y las correspondientes tecnologías. Estas, al igual que la versión dominante de ciencia producto, se reducen al uso de los computadores y las salas de informática y, por supuesto, a la interné. Esta reducción parece manifestar ese desconocimiento de la historia interna y externa del desarrollo del conocimiento científico. Sin embargo, es preciso señalar que en algunas de las ENS estudiadas en esta investigación hay una toma de distancia con respecto a esta concepción dominante.

> Pareceńa ser que la formación histórico-epistemológica, didáctica y pedagógica de los profesores de ciencias de las ENS visitadas, las concepciones acerca de los textos de enseñanza para las ciencias de la naturaleza, no han sido objeto de análisis con base en los resultados de las investigaciones realizadas por quienes se han ocupado de este campo de la didáctica de las ciencias. ya que los resultados de las entrevistas al respecto hablan en favor de una no posición profesionalmente sustentable al respecto. De hecho y hay que subrayarlo, los profesores de ciencias entrevistados señalan que los textos disponibles en el mercado bibliográfico, se ocupan de la socialización de cada una de las ciencias de la naturaleza, desde una perspectiva general y generalizadora de lo que en este proyecto se ha identificado como ciencia producto. Hay que destacar que los profesores de ciencias entrevistados, no aludieron al hecho de que los textos de enseñanza imponen una concepción histórico-epistemológica, didáctica y pedagógica acerca del desarrollo del conocimiento científico y tecnológico.

> Cuando se aludió al problema de la enseñabilidad de las ciencias que hace de cada una de ellas objeto de trabajo en el aula, lo de la transposición didáctica de la actividad cientúfica que practican las respectivas comunidades de especialistas, no apareció en las respuestas a las entrevistas de los profesores de ciencias una conceptualización didáctica crítica.

El problema de la transposición didáctica en la actualidad se remite a disponibilidad en las redes electrónicas de los "originales" a través de los cuales los colectivos de especialistas sometieron a cada una de sus comunidades científicas sus elaboraciones. En consecuencia y de manera preliminar hay que sostener que en Colombia desde finales del siglo XIX, la ciencia que se enseña se haya permeada por ese "pragmatismo oportunista".

\section{Bibliografía}

Barona, J. L. (1994). Ciencia e historia. Debates y tendencias en la historiografía de la ciencia. Valencia: Guada.

Estany, A. (2005). El papel de la historia de la ciencia en los estudios interdisciplinares de la ciencia. En: Historia, filosofía y enseñanza de la ciencia, S. F. Martínez y G. Guillaumin (Comp.), pp. 291 - 303. México: UNAM

Gallego Badillo, R., Pérez Miranda, R., y Rincón Pabón, L. I (2007). Las concepciones que dirigieron la formación de profesores de ciencias en la Escuela Normal Superior de Colombia, desde su inicio hasta 1952 Informe del proyecto DQU-022-07. Bogotá: Universidad Pedagógica NacionalCIUP.

Gallego Badillo, R, Pérez Miranda, R. y Torres de Gallego, L. N. (2004). Formación inicial de profesores de ciencias en Colombia: un estudio a partir de programas acreditados. Ciência \& Educação, Vol. 10, No. 2, $219-234$

Gallego Torres, A. P., Gallego Badillo, R. y Pérez Miranda, R. (2006). ¿Qué versión de ciencia se enseña en el aula? Sobre los modelos científicos y la didáctica de la modelación. Educación $y$ Educadores, Vol. 9, No. 1, 105 - 116 